\title{
Häufige Irrtümer in puncto HzV
}

Zehn Mythen haben die kontroverse Debatte um die AOK-Hausarztverträge mitbestimmt - alle nicht zutreffend, wie Baden-Württembergs Hausärztechef Dr. Berthold Dietsche darlegt:

1. Der Hausarzt wird zum AOK-Hausarzt. Falsch, denn der eingeschriebene Arzt ist Partner der hausärztlichen Vertragsgemeinschaft, die ihrerseits Vertragspartner der AOK ist.

2. Die Therapiefreiheit wird eingeschränkt. Falsch, denn im konkreten Fall entscheidet letztlich immer der Arzt.

3. Die Abrechnung ist zu aufwendig. Falsch, denn neben wenigen Pauschalen gibt es noch eine Reihe von Einzelleistungen.

4. Der Vertrag hält in puncto Honorar nicht das, was er versprochen hat.
Falsch, denn der Fallwert liegt bei 80 Euro. Die werden bei der KV weit unterschritten.

5. Die Einzelleistungen sind in Pauschalen versenkt worden. Falsch. Es kommt auf das Gesamthonorar an, das der Arzt in der $\mathrm{HzV}$ (Hausarztzentrierte Versorgung) erzielen kann; mit welchen Leistungen, bleibt seine freie Entscheidung.

6. Die Vertragssoftware funktioniert nicht und ist zu teuer. Ein Startproblem. Inzwischen werden mehrere arbeitsfähige Systeme angeboten.

7. Die Vertretungsfrage ist nicht klar geregelt. So nicht richtig: Im Regelfall muss ein vertretender Arzt ebenfalls HzV-Arzt sein. Das kann aber im Einzelfall flexibel gehandhabt werden.
8. VERAH lohnt sich nicht. Die Zusatzqualifikation ist zwar teuer mit $4000 \mathrm{Eu}$ ro. Sie rentiert sich aber aufgrund der Zuschläge nach etwa einem Jahr.

9. Wenn der Vertrag nicht funktioniert, steht man als Arzt vor dem Nichts. Falsch, denn der geltende Vertrag läuft bis 2015; lange Kündigungsfrist, Rückkehr ins KV-System ist möglich.

10. Die HzV entwickelt sich nicht weiter. Falsch. Dieses Jahr kommen Neurologen/Psychiater als weitere Facharztgruppen hinzu, sodann Orthopäden. Nach den Arznei- steht nun die Rationalität der Heilmittelverordnungen auf dem Plan, ferner ein System zur Optimierung von Klinikeinweisungen. Darüber hinaus wird der Vertrag evaluiert.

$\mathrm{HL}$.

\section{Bilanz nach drei Jahren mit Hausarztverträgen}

\section{AOK und Hausärzte zeigen: Wettbewerb geht}

\begin{abstract}
Mehr als eine Million eingeschriebene Patienten, fast 3800 teilnehmende Ärzte, eine verbindliche Qualitätssicherung und die koordinierte Behandlung chronisch Kranker - die AOK im Südwesten hat eine Alternative zur Regelversorgung geschaffen.
\end{abstract}

- Von Bürokratie und Honorarwirrwarr gestresste Ärzte, Patienten, die von ihren Ärzten mit Rationierungen verunsichert werden, unnötige Kosten und mindere Qualität als Folge einer unkoordinierten Versorgung - das sind nach Auffassung vieler Experten Schwächen des konventionellen Versorgungssystems.

Erste Vertragspartner der $\mathrm{HzV}$ waren mit dem KV-System unzufriedene Haus-

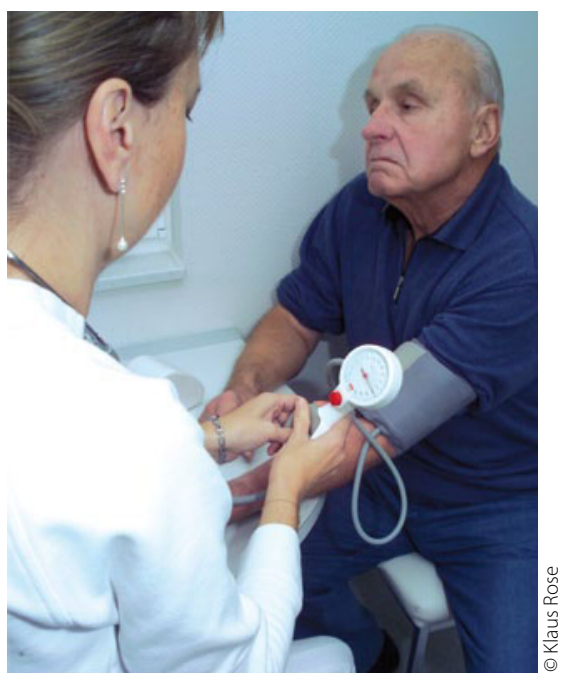

Die Hausarztverträge haben sich inzwischen etabliert. ärzte, Medi folgte sukzessive mit Kardiologen und Gastroenterologen. 73c-Verträge mit Neurologen/Psychiatern sind fast fertig und Verträge mit den Orthopäden sind in Arbeit.

Drei Jahre nach dem Start der Hausarztverträge der AOK wurde beim Hauptstadtkongress Bilanz gezogen. Die Verträge erreichen die Zielgruppe in hohem Maße. 66\% der eingeschriebenen Versicherten sind chronisch krank; 33\% sind es in der konventionellen Versorgung. Das heißt: Ärzte, die in 73b- und $73 c$-Verträgen arbeiten, bewältigen eine besonders hohe Morbiditätslast mit großem Koordinationsaufwand.

Dem gegenüber steht die Chance, die Versorgungsqualität zu verbessern und 